\title{
Advocacy for HIV/TB co-infection collaborative policy and service delivery in India: a civil society perspective
}

\author{
K. Srikanth Reddy ${ }^{1}$, Seema Sahay ${ }^{2}$ \\ ${ }^{1}$ McGill University, Montreal, Canada \\ ${ }^{2}$ National AIDS Research Institute (ICMR), Pune, India
}

\begin{abstract}
Introduction: In the context of evolving policy for human immunodeficiency virus (HIV)/tuberculosis (TB) co-infection in India, the study was conducted to explore civil society perspectives for HIV/TB co-infection collaborative policy and service delivery in the country.
\end{abstract}

Material and methods: Twenty-six in-depth interviews were conducted with purposively selected representatives of advocacy organizations, health activist, positive networks of people living with HIV (PLWHA), and general community. The interviews were audio-recorded, transcribed, coded using QSR NUD*IST software version 6.0, and thematically analyzed. The study was approved by the ethics committee.

Results: The civil society prioritized HIV/TB co-infection as an important public health issue and advocated identifying common indicators for co-infection for better program management. 'Team training concept' for increasing 'antiretroviral therapy coverage' and 'intensified tuberculosis case finding' were reported successfully in Karnataka, and replication of such models of service delivery in other parts of the country was the expectation. Referring to the disparities observed in the national HIV/TB program, the collaborative policy must be inclusive to address co-infection in all high-risk population including intravenous drug users. Principles of 'greater involvement of people with HIV/ acquired immune deficiency syndrome (AIDS)' was advocated for district coordination committees and service delivery, suggesting the possible role of PLWHA as a 'directly observed treatment short course' provider. Advocacy has a significant role in policy decisions if provided with strong evidence base, but communication between research, advocacy, and policy makers remains a challenge.

Conclusions: Advocacy made valuable contributions to national AIDS control program in India. However, advocacy for HIV/TB co-infection policy is still in a nascent stage requiring consultative and inclusive approaches.

HIV AIDS Rev 2018; 17, 3: 259-266 DOI: https://doi.org/10.5114/hivar.2018.80257

Key words: India, advocacy, HIV/TB co-infection, PLWHA, public health policy.

Address for correspondence: Dr. K. Srikanth Reddy, Faculty of Medicine, McGill University, 3640 rue de la Montagne, H3G 2A8 Montreal, Canada, phone: +1 438838 4925, e-mail: srikanth.kondreddy@mcgill.ca
Article history:

Received: 08.06.2017

Received in revised form: 12.02.2018

Accepted: 16.03.2018

Available online: 15.08.2018
International Journal of HIV-Related Problem

HIV \& AIDS

R e v i e w 


\section{Introduction}

Advocacy is a set of targeted actions directed at the decision makers in support of a specific policy [1]. Advocacy can be in several ways, but all of them have a common aim to influence decision-making with the goals of developing, informing or changing policies, establishing and sustaining programs and services for a variety of public issues including health [2]. Public health advocacy is a practice intended to reduce death or disability in groups of people (overall or from a specific cause) and is not confined to clinical settings [3]. The civil society, which is often referred as a 'social sphere' separate from the state and the market, plays a major role in public health advocacy. The civil society sphere constitutes "academic institutions, business forums, clan and kinship circles, consumer advocates, development cooperation initiatives, environmental movements, ethnic lobbies, foundations, human rights promoters, labor unions, local community groups, relief organizations, peace movements, professional bodies, religious institutions, think tanks, women's networks, youth associations, and more" [4].

The human immunodeficiency virus (HIV)/tuberculosis (TB) co-infection is an emergent pandemic. At least onethird of the 36.7 million people living with HIV worldwide are infected with TB and they are 20-30 times more likely to develop TB than those who are not HIV-infected [5]. The co-infection is a major public health problem in many parts of the world, particularly in resource constrained settings with a high burden of both the diseases, such as in Africa and Asia nations [6]. In order to guide the national policies for this dual epidemic, in 2004, the World Health Organization (WHO) developed an interim policy for collaboration between HIV and TB programs for managing the co-infection. The objectives of the interim policy were: 1) to establish the mechanisms for collaboration between $\mathrm{TB}$ and HIV programs; 2) to decrease the burden of tuberculosis in people living with HIV/acquired immune deficiency syndrome (AIDS); and 3) to decrease the burden of HIV in tuberculosis patients [7].

India, an Asian nation, bears the third highest disease burden of HIV-associated TB in the world, posing unprecedented challenges on the public health system in the country [8]. The co-infection cases are being detected through HIV testing of TB patients and intensified case finding at HIV care and treatment centers. Currently, six high HIV prevalence states in India contribute about $75 \%$ of the detection, and concerns are being raised over the existing gaps in detection of co-infection cases particularly in low HIV prevalence states [6]. National HIV/TB co-infection policy in India is evolving [9]. Starting 2001, HIV/TB collaborative activities were implemented in the six high HIV prevalence states. Subsequently, in 2007, the National AIDS Control Organization (NACO) and the Central Tuberculosis Division (CTD) established the first National Frame work of Joint HIV/TB Collaborative Activities, expanding basic HIV/ TB activities to all the states [10]. In 2008, guidelines were changed to implement an 'intensified HIV/TB package' in nine states with the highest HIV prevalence. This also included referral to HIV counseling and testing sites for HIV tests free of charge for all TB patients. Selective referral was continued in the other 26 states in the country [11]. The National Framework for Joint HIV/TB Collaborative Activities was further revised in 2009 to establish uniform guidelines for counselling and testing centers as well as ART centers nationwide, to standardize monitoring and evaluation, and to expand the intensified HIV/TB package to all states by 2012 [12]. Nationwide coverage of the intensified HIV/TB package was achieved in 2012 [6].

Over the years, this policy evolution was guided by international and national advocacy efforts of local research evidences [13]. The main thrust of advocacy efforts was to create widespread awareness among decision makers, community leaders, and media about the dual nature of HIV/TB epidemic, promoting better collaboration and information exchange among TB and HIV programs at the national and regional levels [14]. In this context, we conducted a study in India to explore 'how' the civil society contributed and/or expressed concerns about the HIV/TB co-infection policy development in the country.

\section{Material and methods}

As civil society include a wide range of actors, we conducted in-depth interviews with representatives from people living with HIV/AIDS (PLWHA) networks, representatives of community-based organization (CBOs), non-governmental organization (NGOs), and general community, including vulnerable population for HIV/TB co-infection. We purposively selected the respondents representing different geographic regions (North, South, East, and West) in the country. The 'maximum variation sampling' principle has been utilized to gain rich information on experiences and perspectives from different geographic regions. The respondents were identified based on their familiarity, their involvement in program, and policy advocacy for HIV/TB co-infection.

\section{Data collection}

Twenty-six $(n=26)$ in-depth interviews were conducted with health activists, representatives of advocacy organizations, positive networks of PLWHA, and general community. The profile of the study respondents has been shown in the Table 1. The interviewees were based in five cities of India: New Delhi, Bangalore, Chennai, Mumbai, Kolkata, and Pune. All the interviews were conducted during personal meetings in the interviewee's offices by the first author, who was trained in conducting interviews for qualitative research studies. The interviews were structured around an interview guide (see appendix 1) developed in line with the existing literature [15] and in consultation with senior scientists at National AIDS Research institute (NARI), where this study 
Table 1. Profile of the respondents

\begin{tabular}{l|c|c}
\hline $\begin{array}{l}\text { Participation } \\
\text { number }\end{array}$ & Organizational focus & Experience (years) \\
\hline 1 & Rights-based foundation & 26 \\
\hline 2 & Health focused non-governmental organization & 19 \\
\hline 3 & Positive network & 13 \\
\hline 4 & Pomen positive network & 15 \\
\hline 5 & Puman rights-based organization & 6 \\
\hline 6 & Positive network & 14 \\
\hline 7 & Clinic-based non-governmental organization & 20 \\
\hline 8 & Academic institution & 25 \\
\hline 10 & Positive network & 10 \\
\hline 11 & Women empowerment & 5 \\
\hline 12 & Slum and scheduled population welfare & 23 \\
\hline 13 & People living with HIV/AIDS, women and child development & 20 \\
\hline 14 & Antiretroviral therapy service delivery & 20 \\
\hline 15 & People living with HIV/AIDS and sexually transmitted diseases & 11 \\
\hline 16 & Community development in rural areas & 15 \\
\hline $17-26$ & High-risk populations, caregivers of people living with HIV/AIDS, social workers & As low as 5 years \\
\hline
\end{tabular}

was conducted. A non-structured interview technique was used to facilitate an open discussion and to explore respondent's narratives in depth. On an average the interviews lasted between 60-90 minutes, were audio-recorded in English language, and transcribed verbatim for analysis.

\section{Data analysis}

Data was entered and analyzed in non-numerical data indexing searching and theorizing, NUD*IST software version 6.0. The interview transcripts were coded for emerging themes to describe an integrating, relational idea from the data [16]. Memo writing assisted in defining these codes and linkages between them. The data was thematically analyzed, using Christoffel's public health advocacy framework that involves 3 stages: information, strategy, and action. These stages are conceptually sequential but, in practice, simultaneous. The work at each stage is continually adjusted according to circumstances at the other stages [3]. This framework was used to delineate the roles, information, strategies, and actions being followed and advocated by the civil society for HIV/TB co-infection policy development in the country.

\section{Ethics statement}

The study participants were informed about the objectives of the study, and all the respondents provided written informed consent for participation prior to being inter- viewed. Interviews were recorded on a digital device with the participants' prior permissions [17], and data were stored in encrypted format with restricted access. Care has been taken to ensure anonymity of all individuals cited in this article.

\section{Results}

This study identifies the strategies devised, actions taken, and solutions proposed by the civil society to inform or influence local, state, or national level decision making in the context of dual epidemic of HIV/TB co-infection. Four major themes emerged from the analysis: 1) HIV/TB co-infection: does it need separate treatment; 2) research is an advocacy tool; 3) the interface: providing support, getting support for policy development; 4) civil society advocacy for HIV/TB co-infection policy. These themes are discussed as follows.

\section{HIV/TB co-infection: does it need separate treatment?}

Since the detection of first HIV case in the country, TB co-infection was evident through the clinical history of PLWHA but the focus remained on prevention and treatment of HIV infection. The clinical research demonstrated that there will be high mortality of PLWHA due to TB co-infection, and increasing mortality got momentum in various states of the country over the years. For many years, the public health programs for HIV and TB remained independent of each other, and a lot of emphasis was given only to HIV 
prevention. On the other hand, the increasing morbidity and mortality due to co-infection demanded new policy frameworks for prevention and treatment. As these two programs held different public health mandates, the HIV/TB collaborations did not seem to converge anywhere.

"Two issues, first being annual high incidence of TB among PLWHA compared to HIV-uninfected; second, being highest incidence of TB among IDUs, whether HIVinfected or uninfected. HIV program runs on prevention and treatment as their mandate, whereas TB program mandates on control and effective treatment. The collaboration and co-ordination between these two programs will remain a challenge at field level." (Participant No. 8)

A significant number of deaths among PLWHAs were due to TB, as HIV-infected individuals have higher risk of developing co-infection and thus, the need emerged for early diagnosis and treatment. The respondents opined that there were lack of opportunities for early diagnosis and treatment in public health facilities in different geographic regions of the country. Most of the study respondents, specially from positive networks, expressed displeasure over lack of clear guideline and opportunities to early screening of TB among HIV patients, adding the complexity of the issue. Nevertheless, few respondents were convinced that despite limited opportunities, HIV/ TB co-infection was managed efficiently with the available diagnosis and treatment options. However, the drug resistance to treatment emerged as a persistent challenge for HIV/TB management in the country.

"We have had enough experiences of HIV and TB treatment. However, co-infection is gaining importance in the wake of MDR and XDR TB. We are now advocating for new diagnostic techniques for MDR TB, and the same was agreed upon by the Government of India." (Participant No. 5)

\section{Research is an advocacy tool}

The respondents were of the opinion that advocacy itself is a key step in the policy process assisting in identification of gaps and persuading decision makers to consider those gaps for policy change. One representative from a positive network explained.

"Advocacy is basically a pressure mechanism to change policy as per communities requirements for universal access to quality care." (Participant No. 5)

The advocates also expressed the need for research evidence to bring about any kind of policy change. The importance of research in the policy process was articulated by several advocates as:

"Research is very important for any advocacy effort for policy change. Without research evidence, advocating for policy change is anecdotal".

Research was considered so important for advocacy that 'not having research evidence' could spell failure for advocacy:

"Often, advocacy in our country fails because we are not substantiating with adequate evidence either quantitatively or qualitatively." (Participant No. 8)
Thus, building pressure without strong evidence does not lead to policy change. Hence, strong 'evidence base' as an advocacy tool helps in the policy negotiations. For example, research evidence indicated that ART should be initiated irrespective of CD4 count among eligible HIV/ TB co-infected patients, and this evidence was negotiated for policy change at the appropriate venues such as coordination meetings and technical working group meetings. The success was also being witnessed in free roll out of antiretroviral therapy (ART) in India. Most of the respondents indicated antiretroviral treatment for HIV-infected individuals as success story of advocacy for policy change. They argued that advocacy is a process, which occur over a period of time. There had been advocacy for free roll out of ART at various levels and subsequently, a free roll out of ART program in India came into a reality. One of the study respondents recollected:

"Early in 2001-2002, we demanded treatment for PLWHA and we are now pressurizing to have second line treatment." (Participant No. 7)

The civil society advocates expressed displeasure over some of the policy decisions. Decision makers at times did not listen to advocates and the policy decisions were taken not considering civil society advocacy. The most narrated case was that of targeted interventions (TIs):

"Despite of strong advocacy for not to launch, the Government of India had initiated TI program, eventually leading to human rights violation." (Participant No. 1)

Information, education, and communication (IEC) combines strategies, approaches and methods that enable individuals, families, groups, organizations, and communities to play active roles in achieving, protecting, and sustaining their own health. A HIV/TB co-infection clinical expert said:

"There is no good IEC material for HIV/TB to inform coinfected patients in the entire country." (Participant No 2)

The respondent further shared experiences of best practices, which have programmatic significance as:

"In Karnataka, one of the states of India, several initiatives were undertaken like team training concept, which helped in achieving 60-67\% ART coverage and intensified TB case finding at ART center, translation of 10-point TB tool in vernacular languages to impart HIV/TB coinfection knowledge among patients, minimizing number of visits to ART centers, provision for nutritional support, etc. Now, we are advocating all these provisions to consider for programmatic level." (Participant No 2)

\section{The interface: providing support, getting support for policy development}

The national framework for HIV/TB collaborative activities identified district as the nodal unit for collaboration. The respondents in the study advocated for strong health systems to support co-ordination between the national programs for HIV and TB, as well as, greater involvement of PLWHA in service delivery for the dual epidemic. As there were issues 
related to stigma and disclosure, some of the respondents even suggested the possible role of PLWHA as a DOTS provider. As representatives of positive networks said:

"Considering disclosure issues while treatment, we strongly believe if PLHWA becomes the DOTS provider, he/ she can provide medicines to general community as well as PLWHA, and there will be no disclosure of PLWHA status." (Participant No. 10)

Respondents were of opinion that traditionally community involvement in HIV had been strong, but TB program was lagging in this aspect; there was a need for community involvement for the co-infected. As one respondent said:

"Greater involvement of people living with HIV (GIPA) remained an important aspect in national AIDS program and its service delivery. However, such involvement was not witnessed at district co-ordination for HIV/ TB co-infection to raise issues of patients." (Participant No. 10)

The respondents further emphasized that community participation in advocacy process is essential. But, limited access to community participation in this process was one of the reasons for existing gap in programmatic decision making. As one of the respondents shared the strength of such participation as:

"We were the part of policy formulation and noticed civil society involvement for HIV. However, such involvement was not observed in the case of TB program. Our advocacy helped in building co-ordination mechanism for HIV/ TB co-infection. MDR TB was not a priority earlier and slowly it has become high priority area for the program." (Participant No. 5)

According to the respondents', research, policy, and advocacy are interdependent, and their interface is critical in the policy development process. However, communication gap was evident and need for structured mechanism was voiced by a respondent as:

"The communication is lacking, because we don't have a mechanism with which the whole thing can happen in a formalized way." (Participant No. 8)

In order to bridge this gap, a HIV/TB clinical expert offered potential solutions emphasizing:

"State co-ordination committees incorporate the research institutes as one representative. That will be the best mechanism. Because, already the platform existing, we have to just incorporate some research person and they should very much take the proactive role and tell the policy maker." (Participant No. 2)

\section{Civil society advocacy for HIV/TB co-infection policy}

Civil society act as intermediate layer between the individual and the state that is capable of resolving conflicts and controlling the behavior of members without public coercion. This was illustrated with an example:

"Civil society advocacy more particularly by the rights activists, helped in prioritizing HIV as a serious issue and its treatment, challenging rights of section $377^{1}$ etc." (Participant No. 4)

Public health experts and health activists opined that people in India are not fighting for their rights, especially health rights, resulting in lack of health advocacy. They had also witnessed advocacy in the West as more lobbying-based; however, such lobbying in India is in its nascent stage:

"Active health movements can be noticed in Africa, better supporting systems for health can be noticed in Brazil, whereas in India, we are yet to witness health activism." (Participant No. 4)

The civil society had played a significant role in bringing policy changes. However, the respondents believed in strong network base for policy advocacy, which was not noticed in the case of co-infection. Ideally, such networks need to be well organized and they should operate efficiently to be successful in influencing the policy change or policy development. A well-established networking system would create a supportive and self-sustaining environment for decision making for HIV/TB co-infection:

"HIV/TB co-infection is one area, where positive networks in India is running behind on advocacy front and needs to work proactively." (Participant No. 7)

The policy advocacy was also being done in the case of HIV/TB co-infection. The respondents identified two aspects for policy advocacy in the case of co-infection: 1) Documentation of disease profiles among individuals, which remained a major problem in the country. 2) Strengthening of health systems with research as an integral part of advocacy, policy, and planning. One health activist highlighting the relevance of health systems in the case of co-infection policy said:

"We need to establish such mechanisms, which have po-

litical and legal backing for successful collaborations at ground level." (Participant No. 1)

According to the respondents, HIV/TB collaborative activities between RNTCP and NACP started initially in the year 2001 and since then, the policy is evolving with inputs at various levels. The respondents who were directly or indirectly involved in the evolving HIV/TB collaborative policy, advocated for integrating HIV/TB collaborative services in the general health system in India:

"The important policy decision, which is to be made is that collaborative services should come under the umbrella of district health society, and should be a part of general health system." (Participant No. 2)

The respondents strongly advocated for community involvement in decision making at each level, as the HIV/TB decision making occurs especially at district co-ordination meetings:

"Because communities, who know their issues, are to be involved in national, state, and district HIV/TB coordination planning." (Participant No. 3)

Section 377 of Indian Penal Code (IPC), which came into force in 1862 , defines unnatural offences. It says: "Whoever voluntarily has carnal intercourse against the order of nature with any man, woman, or animal shall be punished with imprisonment for life, or with imprisonment of either description for a term, which may extend to 10 years, and shall also be liable to fine." 


\section{Discussion}

Advocacy brings together the disparate groups to work together for a common goal [18] and advocacy strategies draw from a range of tactics. These can involve "creating and maintaining effective coalitions, the strategic use of news media to advance a public policy initiative and the application of information and resources to effect systemic changes" [19]. It can also involve gathering and presenting an evidence base for desired changes, although it is worth noting that scientific evidence alone is rarely enough to achieve desired political support for public health decision-making.

Over the decade, the HIV/TB co-infection policy significantly evolved, and collaboration between HIV/TB activities including strong management information systems, joint supervision and monitoring, joint capacity building, employing of technology, and optimal use of resources were achieved. However, the collaboration gaps remain in terms of decentralized availability of HIV testing and treatment services [6], implementation challenges such as provision of infrastructure and human resource, and limited community involvement in the implementation of collaborative activities [20].

The study findings describe a range of advocacy aspects for HIV/TB co-infection policy and program in the country. Since HIV fuels the TB epidemic, HIV programs and TB programs share mutual concerns. Hence, prevention of HIV should be a priority for TB control, and TB care and prevention should be priority concerns of HIV/AIDS programs. In India, like in many other high co-infection burden countries, TB and HIV prevention and treatment programs often run as parallel programs. The policies that call for coordination between TB and HIV programs often do not translate into practice at the local level, which was evident from our study. Therefore, the policy advocates for HIV/TB coordination at all levels especially district level, as district is a nodal unit for program collaboration. Such coordination promotes continuity of care for co-infected PLWHA.

The civil society engagement, especially the vulnerable people in the district coordination committees, will provide inputs for decision-making and effective health care service delivery at nodal level. However, the study shows such coordination was not represented or least represented by communities in decision-making as well as service delivery. Similar to this study findings, community-based organizations and advocates are often not included in the development of HIV/TB co-infection policies in Kenya and El Salvador [21]. The reasons for such minimal community involvement may be attributed to the very nature of these two programs in the country. Greater involvement of people living with HIV/AIDS was the central component of the HIV/ AIDS response [22], whereas TB control has traditionally been based to a great extent on the work by epidemiologists, clinicians, and scientists, without the involvement of social scientists, affected communities, and TB patients.

Additionally, the study respondents made a strong reference to evidence-based advocacy that integrates different sectors of research, policy, action groups, clinicians, and practitioners into an analysis to inform advocacy [23]. For example, initiation of ART among HIV/TB co-infected patients irrespective of CD4 cell count within eight-weeks of starting TB treatment [24]. Similarly, in the study, the civil society advocates 'team training concept' for increasing ART coverage and 'intensified TB case finding', which were reported successful in Karnataka and replication of such evidence-based advocacy models for service delivery of HIV/ TB collaborative activities in other parts of the country. On the other hand, this study shows that the decisions against the evidence ended up in uninformed decisions, leading to failure as the decision to launch targeted intervention violated human rights. The study also shows that advocacy prioritizations are temporal, and priorities get changed over time. With the onset of guidelines or frameworks for HIV/ TB collaborative policy, the advocacy emphasis shifted to marginalized population [25], promoting new diagnostic techniques like GeneXpert [26].

The study had limitations. Our research had purposively selected representation from different geographic regions, except the North-Eastern region of the country. The epidemiology suggests that the region has high prevalence rate of HIV infection and so is HIV/TB co-infection, as the co-infection rate is directly proportional to HIV. However, due to resource limitations, the representation from this region was not included in the study.

\section{Conclusions}

Considering the lack of interface between researchers, advocates, and policy makers in India, biomedical policies need evidence-based advocacy. Advocacy, particularly advocacy systems plays a major role in policy development, where systems advocates encourage changes of law, government, service polices, and community attitudes. However, systems advocacy needs strong evidence base to impact policy decisions. For evolving HIV/TB co-infection policy, commissioned and non-commissioned programmatic research studies were conducted to guide state by state intensification of joint HIV/ TB collaborative activities for the prevention and treatment of dual epidemic across the country. The crucial interface with the civil society can materialize by having consultations with them and by paving way to their inclusiveness in decision making. This process might enhance operational success of co-infection policy and program in the country.

\section{Acknowledgements}

We acknowledge the support of the Indian Council of Medical Research (ICMR). First author was supported by an ICMR fellowship, ref. 3/1/3/JRF-2008/HRD -74 (61071). We thank Dr. Paranjape, former Director of the National AIDS Research Institute, for facilitating the study. We sincerely thank the study respondents for their participation for sharing their views and perspectives. 


\section{Conflict of interest}

The authors declare no potential conflicts of interest with respect to the research, authorship, and/or publication of this article.

\section{References}

1. Project P. Networking for Policy Change. An Advocacy Training Manual. Washington: POLICY Project, 1999.

2. WHO. Advocacy guide: HIV/AIDS prevention among injecting users: workshop manual. World Health Organization, UNAIDS, 2004.

3. Christoffel KK. Public health advocacy: process and product. Am J Public Health 2000; 90: 722-726.

4. Scholte JA. Civil society and democracy in global governance. Global Governance 2002; 8: 281-304.

5. Lawn SD, Churchyard G. Epidemiology of HIV-associated tuberculosis Running Head: Epidemiology of TB/HIV. Curr Opin HIV AIDS 2009; 4: 325-333.

6. WHO. Scaling up of collaborative TB/HIV activities in concentrated HIV epidemic settings: a case study from India. WHO, Geneva 2015.

7. WHO. Interim policy on collaborative TB/HIV activities. WHO, Geneva 2004.

8. Seth P. The situation of HIV/M. tuberculosis co-infection in India. Open Infect Dis J 2011; 5: 51-59.

9. Uhler LM, Kumarasamy N, Mayer $\mathrm{KH}$, et al. Cost-effectiveness of HIV testing referral strategies among tuberculosis patients in India. PLoS One 2010; 5: e12747.

10. NACO. National Framework for Joint HIV/TB Collaborative Activities. NACO, New Delhi 2013.

11. Raizada N, Chauhan LS, Khera A, et al. HIV seroprevalence among tuberculosis patients in India, 2006-2007. PLoS One 2008; 3: e2970.

12. CTD. TB India 2010: RNTCP Status Report. CTD, New Delhi 2010.

13. Reddy KS, Sahay, S. Voices of decision makers on evidence-based policy: A case of evolving TB/HIV co-infection policy in India. AIDS Care 2016; 28: 397-400.

14. Kamba GD. Factors contributing to under utilisation of HIV testing services among TB patients in Malawi, 2013.

15. Hanney SR, Gonzalez-Block MA, Buxton MJ, Kogan M. The utilisation of health research in policy-making: concepts, examples and methods of assessment. Health Res Policy Syst 2003; 1: 2.

16. Richards L. Handling qualitative data: A practical guide. Sage, 2014.

17. Grbich C. Qualitative research in health: an introduction. Sage, 1998

18. Gomm M, Lincoln P, Pikora T, Giles-Corti B. Planning and implementing a community-based public health advocacy campaign: a transport case study from Australia. Health Promotion International 2006; 21: 284-292.

19. Johnson S. Public health advocacy. Edmonton, Alberta. Healthy Public Policy-Alberta Health Services, 2009.

20. Agarwal R, Shastri S, Nagaraja SB, et al. TB-HIV: Challenges for Newer Initiatives in India. ARC Journal of AIDS 2016; 1: 1-2.

21. Institute OS. Involving the Community in Responding to TB/HIV. Outcomes of Community-Led Monitoring and Advocacy. Baltimore, 2008.

22. UNAIDS. The Greater Involvement of People Living with HIV (GIPA). UNAIDS, Geneva 2007.

23. Raghavan CP. Advocacy and Communication in Gender and Development: Implications for working with Ethnolinguistic minority Communities. Bangkok, 2010.

24. Tweya H, Ben-Smith A, Kalulu M, et al. Timing of antiretroviral therapy and regimen for $\mathrm{HIV}$-infected patients with tuberculosis: the effect of revised HIV guidelines in Malawi. BMC Public Health 2014; $14: 183$.
25. Dharmadhikari AS, Gupta J, Decker MR, et al. Tuberculosis and HIV: a global menace exacerbated via sex trafficking. Int J Infect Dis 2009; 13: 543-546.

26. Evans CA. GeneXpert - a game-changer for tuberculosis control? PLoS Med 2011; 8: e1001064. 


\section{APPENDIX}

Table 2. Interview guide for civil society

\begin{tabular}{l}
\hline Topic guide for the in-depth interviews with 26 respondents \\
\hline Personal experiences and knowledge about HIV and/or TB, HIV/TB co-infection. \\
\hline Public health importance of HIV/TB co-infection. \\
\hline Examples of civil society advocacy impacting policy decisions of HIV, TB, and co-infection. \\
\hline Research, advocacy and policy interface. \\
\hline Opportunities and challenges of capacity building for civil society advocacy. \\
\hline Role of CBOs/ NGOs in policy and program development. \\
\hline NGO as co-infection health care service providers (referral patterns). \\
\hline Program awareness for HIV/TB collaborative activities. \\
\hline Experiences of HIV/TB program implementation. \\
\hline Role of PLWHA networks in the HIV/TB coordination program activities.
\end{tabular}

